\title{
Brightness Discrimination Following Forebrain Ablation in Fish
}

\author{
Jerald J. Bernstein ${ }^{1}$ \\ Department of Fisheries, University of Michigan, Ann Arbor, Michigan
}

Received October 24, 1960

\begin{abstract}
The effect of forebrain ablation on brightness discrimination in goldnish has been studied. Cardiac deceleration, a conditioned autonomic response, was used as the measure of discrimination in a series of goldfish. Normal and forebrainablated fish were trained to discriminate between two gray stimuli of different brightness. If the experimental animal made this brightness discrimination in thirty-five trials it was subsequently tested on a black and white stimulus pair to see whether the fish was capable of stimulus generalization. If the fish did not make the brightness discrimination, it was trained to discriminate a different set of stimuli to show that it was conditionable. It was found that forebrain ablation did not result in any loss in the ability of these operated fish to make a brightness discrimination. In fact, the operated animals learned the brightness discrimination more rapidly than normal animals. Furthermore, the forebrainless fish were able to generalize to another brightness problem. Control tests were run to substantiate this finding.
\end{abstract}

\section{Introduction}

Identical colors undoubtedly have varying subjective brightness to different animals. Consequently, research in color vision demands proper experimental design in order to establish which factor (hue or brightness) an animal is discriminating (9). Normal goldfish are capable of making a hue discrimination and can also discriminate the brightness parameter from a complex stimulus containing both hue and brightness (5). The neural pathways that mediate these modalities have not been extensively studied. The forebrain of the goldfish is essential for hue discrimination; following forebrain ablation these animals react to colored stimuli on the basis of the brightness characteristics of the stimuli (3). Therefore, the present study was undertaken to ascertain the effect of forebrain

1 Now at the Laboratory of Neuroanatomical Sciences, National Institute of Neurological Diseases and Blindness, U. S. Department of Health, Education, and Welfare, Public Health Service, Bethesda, Maryland. 
removal on a simple brightness discrimination using gray (achromatic) stimuli.

\section{Method}

Apparatus. Goldfish (Carassius auratus Linn.), 8 to 12 inches long, were suspended in the middle of a 40-gallon aquarium. Cardiac electrodes were bilaterally implanted in the experimental fish in order to obtain an electrocardiogram $(E K G),(3,5)$. The stimulus patches, used in the training and testing of the fish, were laminated, 3/4-inch, clear, plastic squares containing patches of Color-aid ${ }^{2}$ art papers (Table 1). The

TABLE 1

Composition of StmMuli

\begin{tabular}{llc}
\hline \multicolumn{1}{c}{ Patch } & \multicolumn{1}{c}{ Source } & $\begin{array}{c}\text { Per cent } \\
\text { reflectance }\end{array}$ \\
\hline Light gray & No. 3 gray paper & 34.0 \\
Dark gray & No. 6 gray paper & 19.5 \\
Black & Color-aid black & 4.0 \\
White & Color-aid white & 83.0 \\
Green frog & Latex lure & 36.0 \\
Tan bug & Latex lure & 17.0 \\
\hline
\end{tabular}

a The per cent reflectance of the stimuli were measured on a Luckiesh-Taylor Brightness Meter using magnesium carbonate as a reference block.

stimuli designated as the tan bug and green frog were latex fly rod lures. The designation "dark" and "light" means that the patches were respectively lighter than, or darker than the gray background against which all stimuli were presented. The background was Color-aid gray No. 5 paper mounted in glass. All stimuli were mounted on Lucite rods for the purpose of presentation to the experimental animals.

Operative Procedure. The forebrain of the experimental fish was removed by aspiration after the cranial cavity was opened. The opening in the brain case was closed by a metal cap. The operation and cap have been described in detail (3).

Animals designated as sham-operated fish were treated as follows: Following anesthesia and opening of the brain case, the fatty tissue was removed and the brain exposed, but no parts were ablated. A cap was then placed on the operated animal. After training, all sham-operated fish were reoperated and converted to forebrainless fish.

2 Color-aid Swatch Book; compiled and coordinated by Sidney Beller, copyright 1948. 
After training and testing, the brains of all forebrain-ablated animals were perfused, stained, and sectioned $(15 \mu)$ according to the pyridine silver technique (6).

Training Procedure. The procedure involved conditioning of the heart rate using electric shock as the unconditioned stimulus and the various stimulus patches as the conditioned stimuli. The cardiac responses to the various stimuli were determined from the EKG. The strength of shock was adjusted individually for each fish by finding the lowest voltage that produced a clearly visible startle response (4-12 volts). In each training or testing series for a given fish, a pair of stimuli was employed. During training one stimulus of the pair (the neutral stimulus) was presented for $10 \mathrm{sec}$ and was never paired with shock. The other (the positive stimulus) was presented for $5 \mathrm{sec}$ and was paired with intermittent shock for the last $2 \mathrm{sec}$ of presentation. One minute was allowed between training trials. During training both stimuli were presented at random until a clear differential cardiac deceleration to the positive stimulus had developed. Checks for cardiac conditioning were run during the training series after each ten conditioning trials and consisted of separate 10 -sec presentations of the positive and neutral stimuli. No shock of course was used during any of these trials.

During test trials both the positive and the neutral stimuli were singly presented for $10 \mathrm{sec}$, without shock, while recording the heart beat of the fish. At this time the test stimuli were always presented in counterbalanced order (ABBA) to make certain that the order of stimulus presentation had not produced temporal conditioning. The time between trials during test periods averaged somewhat longer than 1 min because it was frequently necessary to wait several minutes before the heart rate of the test animals returned to baseline level. During test trials, the heart rate was recorded for $10 \mathrm{sec}$ before and for the $10 \mathrm{sec}$ of the stimulus presentation.

Experimental Design. This experiment tests the ability of forebrainablated fish to make a brightness discrimination. The stimuli used for training and testing are summarized in Table 2. The brightness relationships of the gray stimuli were determined in an earlier experiment in which training and testing sequences established that the goldfish perceived the gray stimuli as lighter and darker than the gray background against which they were presented (5). Of the sixteen fish in this experiment, eight fish were trained with the dark gray as positive and the light gray as neutral. Of the eight fish trained to dark gray as positive, four were normal, one 


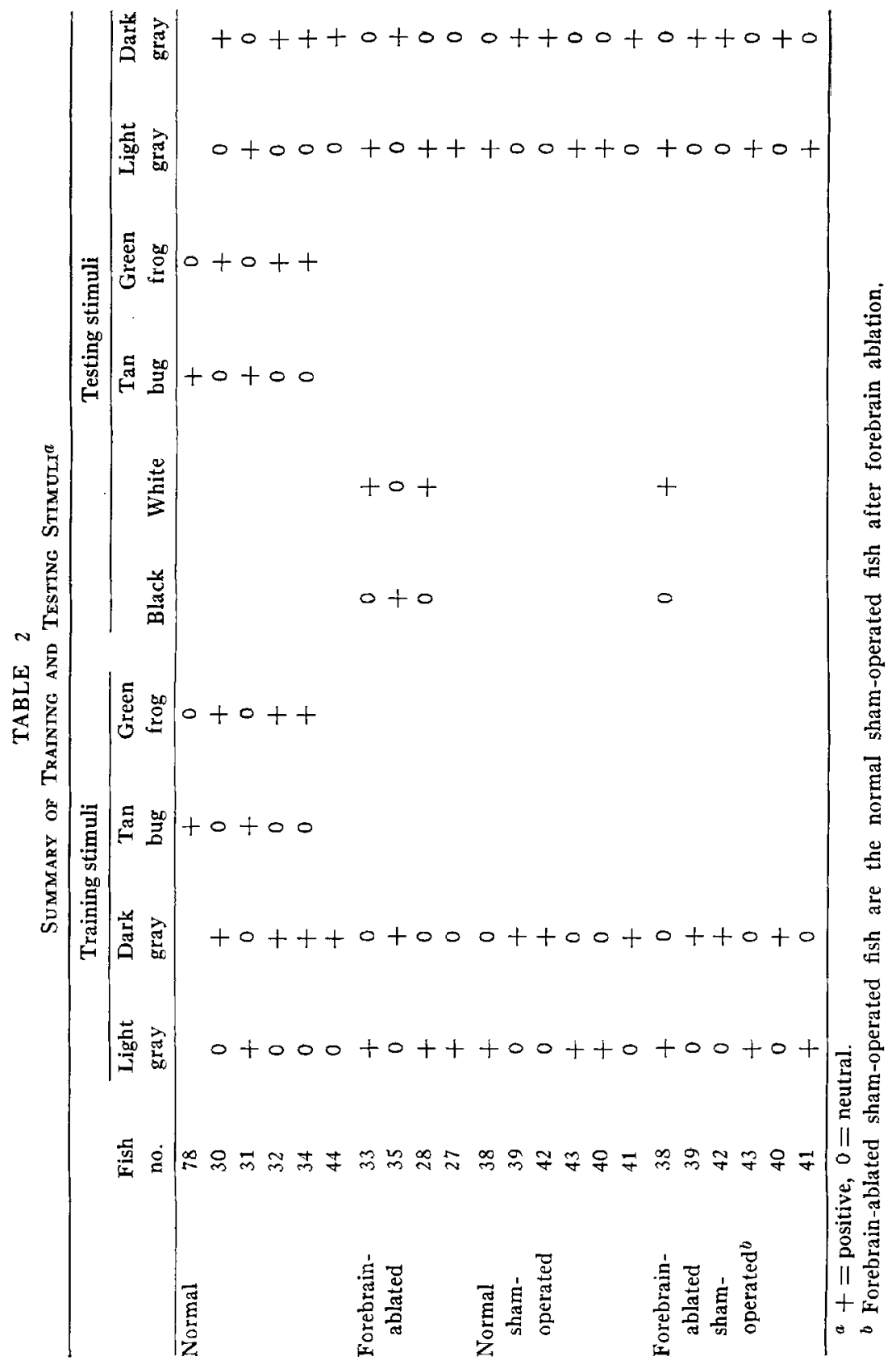


was forebrain-ablated, and three were sham-operated animals. Of the eight fish trained to light gray as positive, two were normal, three were forebrainless preparations, and three were sham-operated animals (Table 2).

In those fish that learned the gray problem within thirty-five trials, additional tests with black and white stimuli were run to test the ability of these fish to generalize to another brightness problem. Of those fish that did not learn the brightness problem by thirty-five trials one of two procedures were followed. If the animal was a normal control, it was trained to discriminate between a green frog and tan bug to make sure it was a conditionable animal. If the animal was a sham-operated fish, its forebrain was removed and its previous training was continued (occasionally the positive and neutral stimuli were reversed).

\section{Results}

Brightness Discrimination in Normal and Sham-Operated Fish. The mean results for twelve fish are shown in Fig. 1. These animals were

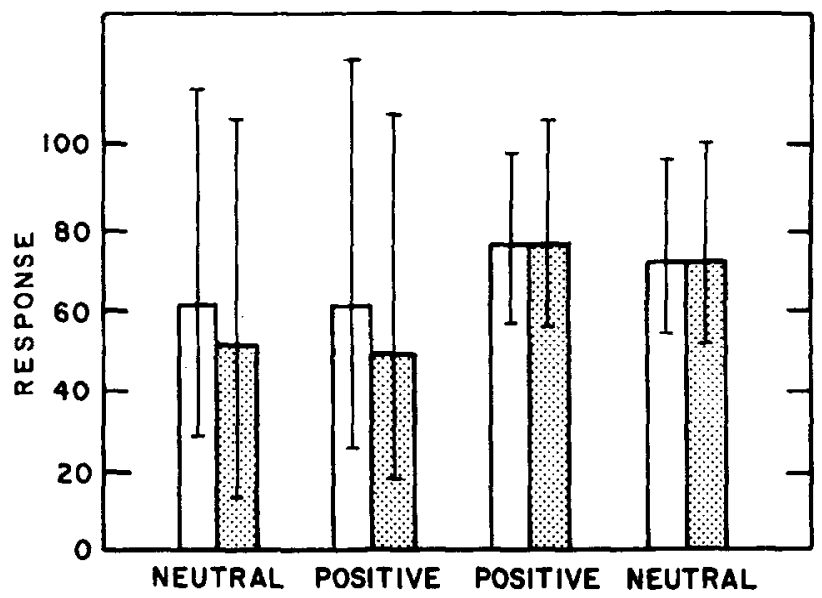

Fig. 1. Mean heart responses and ranges in beats per minute (BPM) for brightness discrimination in twelve normal fish $(p=0.72,11 d . f$.$) . The clear bars represent$ the heart rate $10 \mathrm{sec}$ before stimulus presentation; the stippled bars represent the heart rate during stimulus presentation.

trained to the light gray and dark gray patches (Table 2). The four pairs of bars represent the average of thirty-six test trials from six normal and six sham-operated animals. The results from sham-operated and 
normal animals were statistically indistinguishable; therefore, the two groups were combined. The cardiac responses to the positive stimuli were not significantly different from those to the neutral stimuli $(p=0.72$, 11 d.f.). These data demonstrate that normal and sham-operated animals are not capable of learning (as shown by autonomic conditioning) this relative brightness discrimination within thirty-five training trials.

Control Procedure for Demonstrating Learning Ability in Normal Fish. Five normal animals were trained to discriminate between a tan bug and green frog (Table 2) in order to ascertain whether or not normal fish were capable of any learning. The mean results of the test trials are shown in Fig. 2.

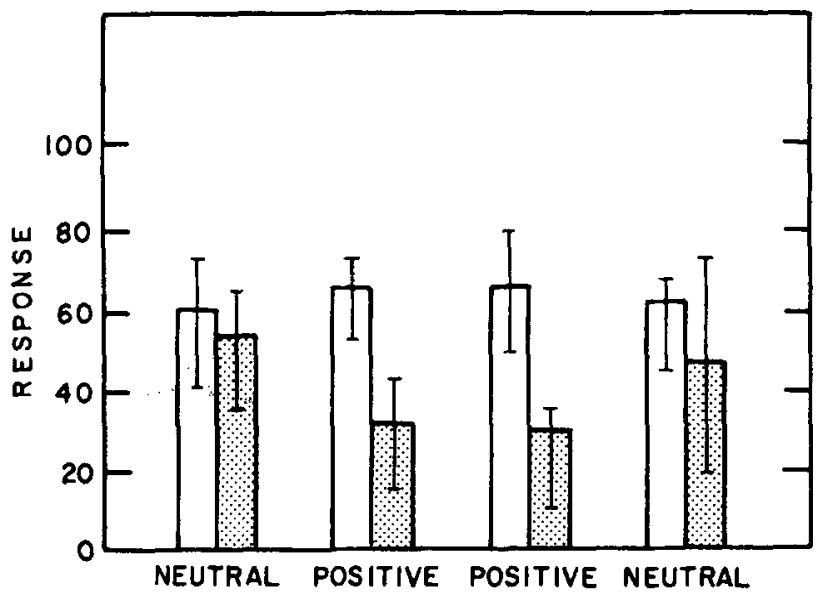

Fig. 2. Mean heart responses and ranges in beats per minute (BPM) for learning in five normal fish $(p=0.02,4$ d.f. $)$. The clear bars represent the heart rate $10 \mathrm{sec}$ before stimulus presentation; the stippled bars represent the heart rate during stimulus presentation.

The cardiac responses to the positive stimuli were significantly greater than to the neutral stimuli $(p=0.02,4$ d.f.). These animals learned the frog-bug problem within an average of eighteen trials. Therefore, it cannot be assumed that they did not make a brightness discrimination within thirty-five trials in the former section because they were unconditionable.

Brightness Discrimination in Forebrain-Ablated Fish. The mean results of the ten fish trained to dark and light gray patches (Table 2) are shown in Fig. 3. The four pairs of bars represent the average of fiftysix test trials. The results from the forebrainless sham-operated and fore- 
brain-ablated fish were statistically indistinguishable; therefore, the two groups were combined.

The forebrainless fish learned the brightness discrimination within thirty-five training trials $(p<0.01,9$ d.f. $)$. Furthermore, all of the sham-operated animals that were trained, ablated, and subsequently re-

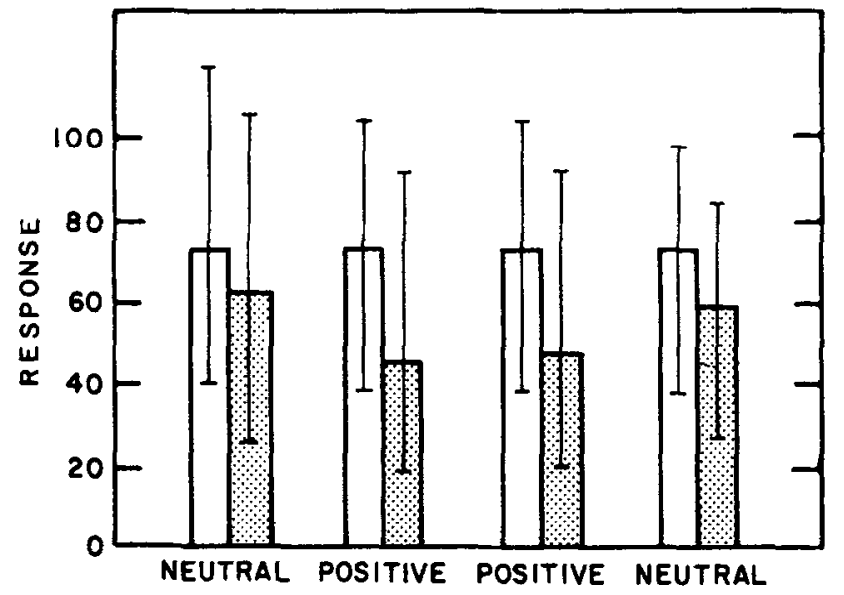

FIG. 3. Mean heart responses and ranges in beats per minute (BPM) for brightness discrimination in ten forebrain-ablated fish $(p=0.01,9$ d.f. $)$. The clear bars represent the heart rate $10 \mathrm{sec}$ before stimulus presentation; the stippled bars represent the heart rate during stimulus presentation.

trained, learned the problem within fifteen trials following forebrain ablation even though they had not learned it in thirty-five trials preoperatively when they served as sham-operated controls. It is interesting to note that the two sham-operated animals in which the positive and neutral stimuli were reversed during postoperative training still learned the new problem in an average of fifteen trials.

Stimulus Generalization in Forebrain-Ablated Fish. Forebrain-ablated fish trained to discriminate between the light gray and dark gray stimuli were found capable of generalizing appropriately to a black and a white stimulus pair. Three forebrainless and one sham-operated forebrainless fish were used in this test of stimulus generalization (Table 2). The mean results of thirty-six test trials are shown in Fig. 4. The cardiac responses to the positive stimuli were significantly larger than those to the neutral stimuli $(p<0.01,3 d . f$.$) . It may be concluded that fore-$ 
brainless animals trained to a relative brightness discrimination can generalize to another brightness problem.

Histological Results. Serial cross sections of the brains of twelve forebrain-ablated fish were examined microscopically. All lesions proved to be alike in extent; the first normal section was the level of the middle of the transverse commissure (Fig. 5). The nucleus preopticus was intact

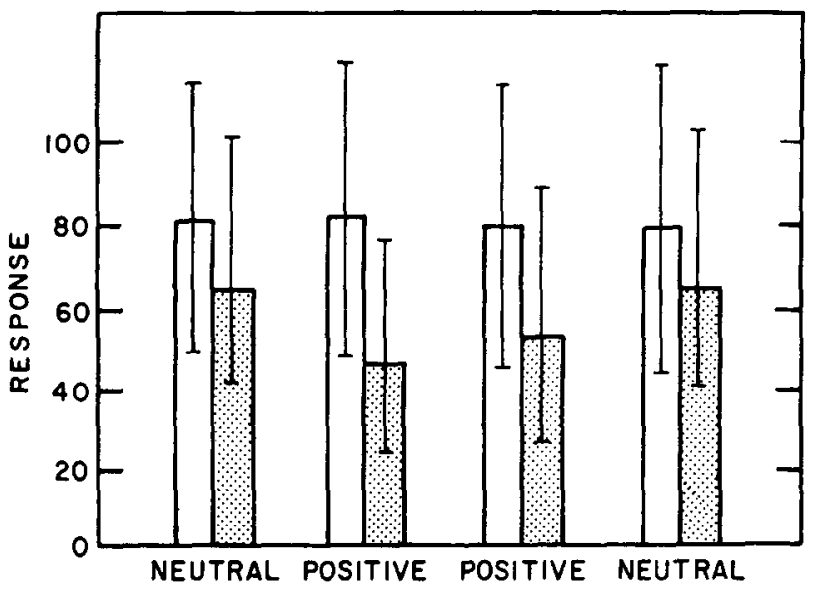

FIG. 4. Mean heart responses and ranges in beats per minute (BPM) for stimulus generalization in four forebrain-ablated fish $(p=0.01,3 d . f$.$) . The clear bars$ represent the heart rate $10 \mathrm{sec}$ before stimulus presentation; the stippled bars represent the heart rate during stimulus presentation.
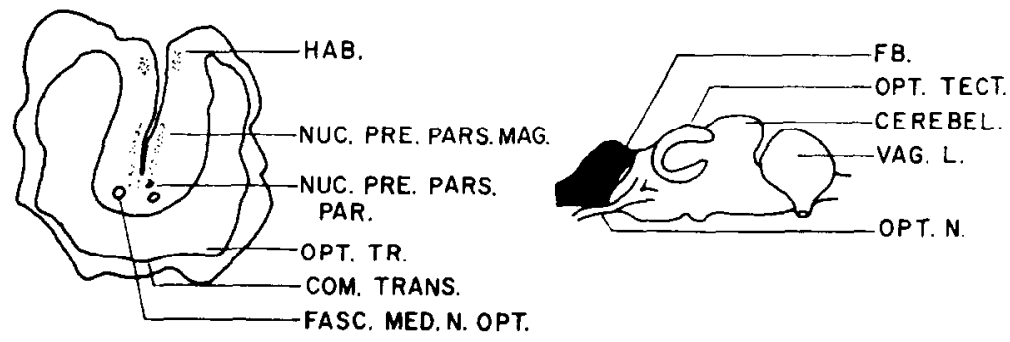

Frg. 5. Reconstructed longitudinal and cross section of the goldfish brain at a level midway through the transverse commissure. Abbreviations: Cerebel., cerebellum; Com. Trans., commissura transversa; Fasc. Med. N. Opt., fasciculus medialis of the optic nerve; FB., forebrain; Hab., habenular nucleus; Nuc. Pre. Pars. Mag., nucleus prcopticus pars magnocellularis; Nuc. Pre. Pars. Par., nucleus preopticus pars parvocellularis; Opt. N., optic nerve; Opt. Tect., optic tectum; Op. Tr., optic tract; Vag. L., vagal lobe. 
throughout its caudal half. The habenular nucleus was intact in all animals examined $(1,2,8)$.

\section{Discussion}

This group of experiments shows the effect of forebrain removal on a simple, brightness discrimination using an arbitrary cutoff point of thirtyfive training trials. It is clear that the forebrain-ablated fish can learn a simple, brightness discrimination with greater ease than can normal fish.

Even in higher animals the lower midbrain visual centers are capable of mediating a brightness discrimination. Rats (7) and dogs (10) can make brightness discriminations after removal of the striate cortex. The ability to make such a discrimination is present to a limited extent in decorticate primates (4). It is not surprising therefore to find forebrainablated ("decorticate") fish possess the ability to make a brightness discrimination.

It is difficult to interpret the improved ability of forebrain-ablated fish to learn a brightness discrimination. The loss in the ability to discriminate hue (3), and conceivably other more complex stimulus parameters as well, rendered the forebrain-ablated fish capable of responding only to the brightness differences in their environment. With visual sensitivity narrowed in such a manner, it would not be surprising if brightness differences in the training stimuli became more conspicuous to the experimental animal.

\section{References}

1. ARIËNS KAPPERS, C. U., The structure of the teleostean and selachian brain. J. Comp. Neurol., 16: 1-109, 1906.

2. ArIËNS Kappers, C. U., F. HUBer, and E. Crosby, "The comparative anatomy of the nervous system of vertebrates, including man." Macmillan, New York, 2 Vols., 1936.

3. Bfrnstrin, J. J., Lass of hue discrimination in forebrain ablated fish. Exptl. Neurol. 3: 1-17, 1961.

4. LASHLEY, K. S, The mechanisms of vision: XVII. Effects of destroying the visual "associative areas" of the monkey. Genet. Psychol. Monographs 37: 107-166, 1948.

5. MCCieary, R. A., and J. J. Bernstein, A unique method for control of brightness cues in study of color vision in fish. Physiol. Zool. 32: 284-292, 1959.

6. Ranson, S. W., The structure of the spinal ganglia and spinal nerves. I. Comp. Neurol. 22: 159-173, 1912.

7. Schwartz, A. A., and S. Clark, Discrimination of intermittent photic stimulation in the rat without its striate cortex. J. Comp. Physiol. Psychol. 17: 233-265, 1957. 
8. Sheidon, R. E., The olfactory tracts and centers in teleosts. I. Comp. Neurol. 22: $177-254,1912$.

9. Walls, G. L., The vertebrate eye and its adaptive radiation. Cranbrook Inst. Sci., Bull. No. 19: 467-472, 1942.

10. WiNG, K. G., The role of the optic cortex of dogs in the retention of learned responses to light: conditioning with light and food. Am. J. Psychol. 60: $30-67,1947$. 